\title{
Incidence, bacteriological profile and antibiotic sensitivity pattern of neonatal sepsis in a tertiary health facility in Abuja, North-central Nigeria.
}

Olutunde Oluyinka

West Suffolk Hospital

Kareem I. Airede

University of Abuja Teaching Hospital

Kudi E. Olateju

University of Abuja Teaching Hospital

Obaro K. Stephen

University of Nebraska Medical Center

Nosakhare Izevbigie

University of Abuja

Kenechukwu K. Iloh

University of Nigeria Teaching Hospital

Obianuju Igbokwe

University of Nigeria Teaching Hospital

Chidiebere Donatus Ignatius Osuorah ( $\sim$ chidi.osuorah@gmail.com)

MRC Laboratories The Gambia

Research article

Keywords: neonatal sepsis, mortality, cross-sectional study, antibiotic sensitivity

Posted Date: January 23rd, 2020

DOI: https://doi.org/10.21203/rs.2.21673/v1

License: @ (i) This work is licensed under a Creative Commons Attribution 4.0 International License. Read Full License 


\section{Abstract}

Background: Neonatal sepsis is commonly caused by bacteria in the first 28 days of life. If prompt management is not instituted, it could lead to death within hours of onset. Due to diagnostic limitation in developing settings, prompt laboratory identification of causative organism is usually a challenge. To prevent mortality, clear knowledge of bacteria and their antibiotics sensitivity pattern is important for prompt empirical treatment.

Methods: This prospective and cross-sectional study enrolled 339 newborns and were admitted for probable sepsis to the special care unit of the university of Abuja Teaching Hospital. Socio-demographic profile and Blood culture was done from every enrolled newborn using BACTEC 9050. The pattern of the clinical features, incidence, bacteriological profile and antibiotic sensitivity pattern of newborns with confirmed neonatal sepsis were documented.

Results: A total of 645 newborn were admitted for probable sepsis during the study period based on clinical features and initial laboratory work-up. Forty-six of the 645 newborns (46/645) had laboratory confirmed sepsis based on blood culture resulting in a neonatal sepsis incidence rate of 71.3 (95\%Cl 50.7-91.9) per 1000 admitted newborn. Seventeen of the 46 confirmed sepsis cases were among the 1322 newborns delivered within the study facility during the study period giving an in-hospital neonatal sepsis incidence rate of 12.9 (95\% Cl 6.7-19.0) per 1000 live birth. Amongst the 46 babies with positive blood culture, $27 / 46(58.7 \%)$ had normal white cell count while the remaining 19/46 (41.3\%) had abnormal results. In all, 52 counts of bacteria categorized into 11 bacteria species were isolated from the 46 positive blood cultures. Enterococcus spp and streptococcus species were the commonest gram-positive while Escherichia coli and Chryseomonas luteola were the commonest gram-negative bacteria isolates. Imipenem (all bacterial isolates except Vibrio fluvialis), Augmentin (Streptococcus spp, Staphylococcus spp, Escherichia spp, Enterococcus spp, Klebsiella spp, Moraxella catarrhalis, Acinetobacter baumannii), Vancomycin (Streptococcus spp, Staphylococcus spp, Escherichia spp, Enterococcus spp), and Ofloxacin (all except Vibrio fluvialis and Citrobacter freundii) had the widest coverage of bacteria isolated from newborn with sepsis.

Conclusion: Sepsis in newborn is still prevalent in our environment and compared to previous documented isolates and sensitivity pattern, the bacteria causes, and their antibiotic sensitivity patterns appears to be changing.

\section{Background}

Neonatal sepsis is a life threatening emergency and any delay in treatment may result to septic shock and death. ${ }^{1}$ According to a 2006 report of the World Health Organization, it is estimated that 1.6 million deaths occur globally every year due to neonatal sepsis and it is responsible for 30 to $40 \%$ of all neonatal deaths occurring in developing countries. ${ }^{2-4}$ Nigeria ranks number one in annual newborns death in Africa and second highest in the world. ${ }^{5}$ The neonatal mortality in Nigeria stands at 33/1000 live births. ${ }^{6}$ In developing countries, bacterial pathogens are the most common cause of neonatal sepsis, ${ }^{7}$ causing a wide variety of infections including meningitis, pneumonia, urinary tract infection and sepsis. ${ }^{3,5}$ These infections can run a rapid course with death occurring in less than 24 hours, if prompt effective empirical treatment is not instituted. ${ }^{3,8}$ The development of effective empirical antibiotic protocols depend on the knowledge of the prevailing bacterial pathogens in that locality. In the neonatal period, it has been documented that the commonest blood-culture isolates in

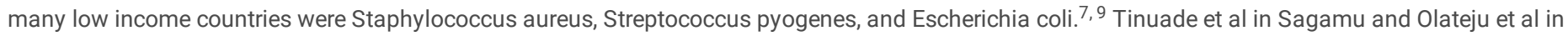
Gwagwalada, described Klebsiella specie and coagulase-negative Staphylococci featuring prominently in Nigeria. ${ }^{10,11}$ The spectrum of organisms that cause neonatal sepsis changes over time and varies from region to region, even in the same center from time to time. ${ }^{3,10}$ These organisms have also developed increasing multi-drug resistance over the last two decades. ${ }^{3}$ Therefore, current knowledge of center specific pattern of bacterial isolates and their antimicrobial susceptibility pattern is useful for prompt management of patients. The present study was undertaken to highlight the local pattern of bacterial isolates in neonates and their antimicrobial sensitivity in a tertiary care hospital in Abuja.

\section{Methods}

Study design and area

This prospective and cross-sectional study was conducted over an 8-month period in 2014 at the Special Care Baby Unit (SCBU) of the University of Abuja Teaching Hospital (UATH). The UATH is a 350-bed tertiary hospital that runs primary and secondary in addition to tertiary health care services. The hospital is in Gwagwalada Area Council of the Federal Capital Territory (FCT), Abuja; North Central region of Nigeria. ${ }^{12}$ It is the main referral centre for neonatal care in north-central region. UATH has annual deliveries of 2,500 babies and an average annual SCBU admission of 968 babies using the year 2012 records. The SCBU is 30-bedded unit with separate inborn and outborns sections manned by a neonatologist, four senior and junior residents each, six house officers and twenty nurses with further training in neonatology. One thousand, three hundred and twenty-two (1322) newborns were delivered in the center during the study period.

Management overview of neonatal sepsis in UATH

On presentation to the SCBU, historical assessment and detailed physical examination are done. After collection of the relevant samples, the subjects are commenced on empiric antibiotics of amoxicillin/clavulanic acid at $15 \mathrm{mg} / \mathrm{kg}$ body weight and gentamicin at $2.5 \mathrm{mg} / \mathrm{kg}$ body weight per dose every 12 hours. Necessary changes are made based on clinical response and/or result of blood culture and sensitive test. Sometimes, third generation cephalosporin mostly cefotaxime and ceftazidime are used for severe infections and in babies who presented with relevant clinical features and/or clinical examination suggestive of meningitis. Other supportive measures where necessary are also initiated.

Sample size determination and sampling technique 
The sample size was calculated using the Cochrane formula, ${ }^{13}$ based on a prevalence of neonatal sepsis from a previous, ${ }^{11}$ a confidence level of $95 \%$ and a level of precision estimated to be $\pm 5 \%$ of the assumed prevalence in addition to a non-response rate of $10 \%$. This resulted to a minimum sample size of 331.Consecutive neonates who met the inclusion criteria were enrolled into the study. The inclusion criteria included neonates admitted to the SCBU with features suggestive of sepsis (such as fever, jaundice, lethargy, poor suck etc) and those whose parents/guardians gave consent for enrolment of their newborn in the study. Excluded from this study were newborns with major congenital malformations (this was to avoid mortality not directly linked to sepsis) and neonates who have had administration of antibiotics for more than 24 hours, prior to presentation to the hospital.

Blood and data collection method

All the neonates that were enrolled into the study had their blood specimens drawn for culture before treatment was initiated or latest within 12 hours of admission. The skin over the site of blood collection was cleaned thoroughly with $70 \%$ alcohol, allowed to dry and cleaned with Povidone iodine for two minutes before blood sample was collected. Two millilitres of blood were drawn by trained assistants from a peripheral vein. One millilitre was for blood culture, and the other for complete blood count (CBC). A structured questionnaire was used to collect relevant information for each newborn. The questionnaire was pre-tested at Federal Staff Hospital, Jabi in Abuja. It was then analysed for completeness and ease of completing the questionnaire. The neonatal information collected included age, sex, weight on admission, place of delivery, and specific clinical features such as fever, jaundice, hypothermia, hyperthermia, poor skin colour, respiratory distress, feed intolerance, bleeding diathesis, and abdominal distension etc.

Measures and definition of term

i. Weight was measured using a standardized Bassinet scale, Salter ${ }^{\mathrm{TM}}$ Model 180 , made in England, with a sensitivity of $0.05 \mathrm{~kg}$ and calibrated in $0.1 \mathrm{~kg}$. Before placing the baby in the weighing scale, the scale was readjusted to zero each time for a new reading. All clothing was removed including the diaper, in order not to affect the weighing scale reading.

ii. Length of each baby enrolled in to the study, was measured using inelastic tape measure, by placing the baby on a hard surface, lying supine and the lower limbs fully stretched.

iii. Occipito-frontal circumference (OFC) was measured using inelastic tape measure. The frontal and occipital bony prominences were identified before measurements were taken using an inelastic tape measure.

iv. Gestational age of each baby was determined using modified Ballard score. ${ }^{14}$

v. The newborns family's Socio-economic status was determined using Olusanya's social economic classification. ${ }^{15}$

vi. Neonatal sepsis for the sake of this study was defined as blood stream infection with positive blood culture result.

Laboratory methods

Blood specimen bottles collected from newborns admitted to the SCBU for probable sepsis were put within one hour of collection into BACTEC 9050 (BectonDickinson $^{\text {TM }}$ New Jersey, USA) automated system, by a trained dedicated microbiologist to the BACTEC laboratory. It was then monitored for growth by the microbiologist, as flagged by the machine, every 24 hours, for maximum of 5 days, when the bottles were expelled by the automated machine. The BACTEC 9050 system, which accommodates 50 test vials, features an extremely small footprint (only 4 1/4 square feet of tabletop needed, and no external computer required). ${ }^{16}$

For gram staining procedure, a single colony was picked and emulsified in a drop of normal saline on a clean glass slide and allowed to dry. The slide was then fixed with heat, stained with crystal violet for one minute, followed by procedure mordant with Lugo's iodine solution for 30 seconds. The slide was then washed with water. The process of decolourisation was carried out with acetone for one to two seconds before washing with water. Counter-staining was with safranin, added for two minute and later washed with water and allowed to air dry. The slide was then examined microscopically. Gram positive bacteria (GPB) were presumed if the identified pathogen appeared dark purple, while a red colour suggested Gram negative bacteria (GNB).

Antimicrobial susceptibility testing was done using modified Kirby-Bauer disc diffusion method, ${ }^{17}$ using multidisc antibiotics (Oxoid Ltd, Hampshire, UK), as described by the Clinical Laboratory Standards. The degree of inhibition of bacterial growth around the antibiotics in the impregnated discs was noted and the result was reported as sensitive or resistant. For determining sensitivity, the following anti-microbial discs were used; Ampicillin $10 \mu \mathrm{g}$, Ceftazidime $30 \mu \mathrm{g}$, Augmentin (Amoxicillin $20 \mu \mathrm{g}$; Clavulanic acid $10 \mu \mathrm{g}$ ), $30 \mu \mathrm{g}$, Vancomycin $30 \mu \mathrm{g}$, Cefotaxime $30 \mu \mathrm{g}$, Ceftriaxone $30 \mu \mathrm{g}$, Gentamicin10 $\mu \mathrm{g}$, ofloxacin $5 \mu \mathrm{g}$ and Imepenam10 $\mu \mathrm{gm}$.

A complete blood count ( $\mathrm{CBC}$ ) with white blood cell differential count was done for each subject. The complete blood count was processed in the general laboratory of University of Abuja Teaching Hospital. The CBC sample bottle was placed in the BC- 3200 autolizer machine Mindray. ${ }^{\mathrm{TM}}$ The machine extracts the required volume of blood for auto analysis and then displayed the result on the monitor. Other investigation done included serum bilirubin estimation for babies with visible jaundice, while serum electrolytes, urea, creatinine and glucose were also evaluated in babies with perinatal asphyxia and neonatal seizures. For the sake of this study, normal white cell count on CBC was defined as cell count between $9-30,000 / \mathrm{mm}^{3}$ (or $9-30 \times 10^{9} / \mathrm{L}$ ). Values above or below this range were considered abnormal.

Data analysis

The raw data were inputted into the Excel spread sheet and analysed using the Statistical Package for Social Sciences (SPSS) version 20 Microsoft USA. Frequency tables were generated for simple proportions and descriptive analysis. 


\section{Results}

Characteristics of newborns enrolled for study

Figure 1 shows a summary of the recruitment process. A total of 645 newborns were admitted to the SCBU during the study period. About two-thirds (474) of the 645 admitted were born within the study center while the remainder (177) were referred to it (Fig. 1). After initial review, 339 of the 645 admitted newborns were enrolled with a presumptive diagnosis of neonatal sepsis. Table 1 shows the socio-demographic characteristics and anthropometric indices of newborns enrolled. About one-third, 110 (32\%) of the enrolled newborns were $\leq 1$ day, 58 (17\%) were $2-7$ days old while $171(51 \%)$ were more than 7 days old. The mean age of babies enrolled was $4.2 \pm 1.6$ days. There were more male enrollees $196(58 \%)$ compared to female $143(42 \%)$. Majority ( $81 \%)$ of the admitted newborn were discharged from the SCBU alive after full recovery, 15 (4\%) left against medical advice following request by parent/caregiver while 49 (15\%) of the 339 newborns died while on admission. Eight of the 49 recorded mortality had sepsis confirmed on blood culture. Other characteristics of enrolled newborns are shown in Table 1.

Table 1

Characteristics of newborns admitted to the SCBU of the UATH between September 2013 to

April 2014

\begin{tabular}{|c|c|c|c|}
\hline Newborn variables & $\begin{array}{l}\text { Frequency } \\
\text { n (\%) }\end{array}$ & Newborn variables & $\begin{array}{l}\text { Frequency } \\
\mathrm{n}(\%)\end{array}$ \\
\hline Age at Presentation & $n=339$ & Gestational at delivery & $n=338$ \\
\hline$\leq 1$ day & $110(32)$ & Pre-term (<37 weeks) & 109 (33) \\
\hline 2-7 days & $58(17)$ & Term (37-40 weeks) & $211(62)$ \\
\hline$>7$ days & $171(51)$ & Post-term (> 40 weeks) & $18(5)$ \\
\hline Sex & $n=339$ & Head circumference & $n=295$ \\
\hline Male & $196(58)$ & $<33 \mathrm{~cm}$ & $99(34)$ \\
\hline \multirow[t]{2}{*}{ Female } & $143(42)$ & $33-37 \mathrm{~cm}$ & $169(57)$ \\
\hline & & $>37 \mathrm{~cm}$ & $27(9)$ \\
\hline Place of delivery & $n=339$ & & \\
\hline Inborn & 168 (49) & Length at birth & $n=268$ \\
\hline \multirow[t]{2}{*}{ Outborn } & $171(51)$ & $<45 \mathrm{~cm}$ & $118(44)$ \\
\hline & & $\geq 45 \mathrm{~cm}$ & $150(56)$ \\
\hline Mode of delivery & $\mathrm{n}=339$ & & \\
\hline Spontaneous Vertex \pm assisted & $214(63)$ & Birth weight & $n=337$ \\
\hline \multirow[t]{2}{*}{ Operational (Caesarean section) } & $125(37)$ & $<2.5 \mathrm{~kg}$ & $148(44)$ \\
\hline & & $2.5-4.0 \mathrm{~kg}$ & $179(53)$ \\
\hline Maternal education & $n=339$ & $>4.0$ & $10(3)$ \\
\hline$\leq 6$ years & $67(22)$ & & \\
\hline $7-12$ years & $118(40)$ & Feeding history & $\mathrm{n}=339$ \\
\hline \multirow[t]{2}{*}{$>12$ years } & $114(38)$ & Breastmilk only & $235(69)$ \\
\hline & & Infant formula \pm breastmilk & $104(31)$ \\
\hline Maternal socio-economic class & $\mathrm{n}=339$ & & \\
\hline Low & $171(51)$ & Outcome & $n=339$ \\
\hline Middle & $58(17)$ & Survived & $275(81)$ \\
\hline \multirow[t]{2}{*}{ High } & $110(32)$ & Died & $49(15)$ \\
\hline & & Left against medical advice & $15(4)$ \\
\hline
\end{tabular}

Incidence rate and clinico-laboratory presentation of neonates with sepsis

Forty-six of the 339 enrolled had bacterial isolates on blood culture. Since 645 newborns were admitted during this period, this resulted to a neonatal sepsis incidence rate of 71.3 (95\% Cl 50.7-91.9) per 1000 admitted newborn. Seventeen of the 46 confirmed sepsis cases were among the 1322 newborns delivered within the study center during the period of study giving an in-hospital neonatal sepsis incidence rate of 12.9 (95\% Cl 6.7-19.0) per 1000 live birth. Twenty- 
seven of the 46 newborns with confirmed sepsis (58.7\%) had white cell count within normal range while the remaining 19 (41.3\%) had abnormal white cell count. Other abnormalities encountered on CBC included anaemia (22.0\%) and polycythaemia (3.0\%).

Bacterial profile and antibiotic sensitivity pattern in newborns admitted for sepsis

In all, 52 counts of organism categorized into 11 bacteria species were isolated among the 46 newborns with blood culture confirmed sepsis. Of these, 28 $(53.9 \%)$ were gram positive bacteria while the remainder, 24 (46.1\%) were gram-negative organisms. Table 2 shows the species of bacteria isolates on blood culture. Enterococcus 16/52 (30.8\%) and streptococcus species 9/52 (17.35) were the commonest organisms isolated from the newborns. Other encountered organisms included Escherichia coli 6/52 (11.5\%), Chryseomonas luteola 5/52 (9.6\%), Klebsiella species 4/52 (7.7\%) Acinetobacter baumanii 3/52 (5.8\%) and staphylococcus species 3/52 (5.8\%). Less commonly cultured bacteria included Pseudomonas species 1/52 (1.9\%), Citrobacter freundii 1/52 (1.9\%) and Vibro fluvialis 1/52 (1.9\%). The different bacterial isolates stratified by selected newborn parameters are summarized in Table 2. For gram-positive organisms, Enterococcus septicemia were seen more in males, late onset sepsis ( $\geq 72$ hours) and neonates born outside the study facility while streptococcus septicemia was more in females, early onset sepsis and outborns. Staphylococcus septicemia was seen more in males, newborns with early onset sepsis, those with birth weight $\geq 2.5 \mathrm{~kg}$ and those delivered within the study facility. Correspondingly, for gram-negative organisms, Escherichia coli was isolated more in males,

newborns that developed sepsis within 72 hours of life, $<2.5 \mathrm{~kg}$ and outborns while Klebsiella spp (female, $\geq 2.5 \mathrm{~kg}$ and inborn) and Pseudomonas spp (male, $<25 \mathrm{~kg}$, EOS and outborns) showed almost reverse infectivity patterns with newborn parameters. Other isolated organisms were almost evenly distributed among the categories of newborns. There were no significant differences in newborns socio-demographic characteristics and bacteria isolates pattern seen (Table 3).

Table 2

Blood culture bacterial isolates stratified by some selected clinical and demographic parameters of newborns admitted for sepsis to the SCBU of the UATH between September 2013 to April 2014

\begin{tabular}{|c|c|c|c|c|c|c|c|c|c|c|c|c|}
\hline \multirow[t]{3}{*}{ Bacterial isolates } & \multicolumn{12}{|c|}{ Newbom parameters } \\
\hline & \multicolumn{3}{|c|}{ Gender } & \multicolumn{3}{|c|}{ Time of onset after birth } & \multicolumn{3}{|c|}{ Birth weight } & \multicolumn{3}{|c|}{ Place of birth } \\
\hline & Male & Female & Total & $<72$ hrs. & $\geq 72$ hrs. & Total & $<2.5 \mathrm{~kg}$ & $\geq 2.5 \mathrm{~kg}$ & Total & Inborn & Outborn & Total \\
\hline \multicolumn{13}{|l|}{ Gram positive organisms } \\
\hline Enterococcus faecalis & 8 & 6 & 14 & 5 & 9 & 14 & 7 & 7 & 14 & 5 & 9 & 14 \\
\hline Streptococcus species & 4 & 5 & 9 & 6 & 3 & 9 & 4 & 5 & 9 & 3 & 6 & 9 \\
\hline Staphylococcus aureus & 2 & 1 & 3 & 3 & 0 & 3 & 0 & 3 & 3 & 2 & 1 & 3 \\
\hline Enterococcus cloaca & 1 & 0 & 1 & 1 & 0 & 1 & 1 & 0 & 1 & 1 & 0 & 1 \\
\hline Enterococcus sakazakii & 1 & 0 & 1 & 1 & 0 & 1 & 0 & 1 & 1 & 0 & 1 & 1 \\
\hline \multicolumn{13}{|l|}{ Gram negative organisms } \\
\hline Escherichia coli & 4 & 2 & 6 & 4 & 2 & 6 & 4 & 2 & 6 & 2 & 4 & 6 \\
\hline Chryseomonas luteola & 4 & 1 & 5 & 3 & 2 & 5 & 2 & 3 & 5 & 2 & 3 & 5 \\
\hline Klebsiella species & 1 & 3 & 4 & 2 & 2 & 4 & 1 & 3 & 4 & 3 & 1 & 4 \\
\hline Acinetobacter baumanii & 1 & 2 & 3 & 2 & 1 & 3 & 0 & 3 & 3 & 0 & 3 & 3 \\
\hline Pseudomonas species & 2 & 0 & 2 & 0 & 2 & 2 & 2 & 0 & 2 & 0 & 2 & 2 \\
\hline Citrobacter freundii & 0 & 2 & 2 & 1 & 1 & 2 & 1 & 1 & 2 & 1 & 1 & 2 \\
\hline Vibro fluvialis & 1 & 0 & 1 & 0 & 1 & 1 & 1 & 0 & 1 & 0 & 1 & 1 \\
\hline Moraxella catarrhalis & 1 & 0 & 1 & 1 & 0 & 1 & 0 & 1 & 1 & 1 & 0 & 1 \\
\hline Total & 30 & 22 & 52 & 29 & 23 & 52 & 23 & 30 & 52 & 21 & 32 & 52 \\
\hline
\end{tabular}


Bacteria isolates and patterns of antibiotic responses among newborns presenting with sepsis to the SCBU of the UATH between September 2013 to $\mathrm{Al}_{\mathrm{I}}$

\begin{tabular}{|c|c|c|c|c|c|c|c|c|c|c|c|c|c|c|c|c|c|}
\hline \multirow{2}{*}{$\begin{array}{l}\text { Bacteria isolate in } \\
\text { Blood }\end{array}$} & \multicolumn{17}{|c|}{ BACTERIAL ANTIBIOGRAM (\%) } \\
\hline & AM & $\mathrm{AU}$ & $\mathrm{CH}$ & $\mathrm{CL}$ & $\mathrm{CL}_{2}$ & $\mathrm{CO}$ & CE & $\mathrm{CE}_{2}$ & $\mathrm{CE}_{3}$ & $\mathrm{CE}_{4}$ & $\mathrm{Cl}$ & ER & GE & $\mathrm{IM}$ & LE & ME & OF \\
\hline Streptococcus spp & 10.0 & 80.0 & 20.0 & 0.0 & 60.0 & 0.0 & 80.0 & 90.0 & 20.0 & 0.0 & 0.0 & 30.0 & 0.0 & 60.0 & 0.0 & 0.0 & 40.0 \\
\hline Staphylococcus spp & 0.0 & 40.0 & 60.0 & 20.0 & 60.0 & 0.0 & 0.0 & 0.0 & 0.0 & 20.0 & 0.0 & 40.0 & 20.0 & 60.0 & 0.0 & 0.0 & 20.0 \\
\hline Escherichia spp & 14.0 & 50.0 & 65.0 & 0.0 & 14.0 & 7.0 & 21.5 & 21.5 & 14.0 & 0.0 & 7.0 & 14.0 & 21.5 & 50.0 & 7.0 & 7.0 & 14.0 \\
\hline Pseudomonas spp & 0.0 & 0.0 & 0.0 & 0.0 & 0.0 & 0.0 & 0.0 & 0.0 & 100 & 0.0 & 100 & 0.0 & 0.0 & 100 & 0.0 & 0.0 & 100 \\
\hline Enterococcus spp & 5.0 & 15.5 & 18.5 & 0.0 & 5.0 & 0.0 & 2.5 & 2.5 & 0.0 & 0.0 & 2.5 & 5.0 & 5.0 & 15.5 & 5.0 & 5.0 & 2.5 \\
\hline Klebsiella spp & 0.0 & 25.0 & 50.0 & 0.0 & 0.0 & 0.0 & 75.0 & 75.0 & 25.0 & 0.0 & 0.0 & 0.0 & 25.0 & 100 & 0.0 & 0.0 & 50.0 \\
\hline Moraxella catarrhalis & 100 & 100 & 100 & 0.0 & 0.0 & 0.0 & 100 & 0.0 & 100 & 0.0 & 100 & 0.0 & 100 & 100 & 0.0 & 0.0 & 100 \\
\hline Vibrio fluvialis & 0.0 & 0.0 & 100 & 0.0 & 0.0 & 100 & 0.0 & 100 & 0.0 & 0.0 & 0.0 & 0.0 & 100 & 0.0 & 0.0 & 0.0 & 0.0 \\
\hline $\begin{array}{l}\text { Acinetobacter } \\
\text { baumannii }\end{array}$ & 0.0 & 33.3 & 0.0 & 0.0 & 0.0 & 0.0 & 0.0 & 0.0 & 66.7 & 0.0 & 100 & 0.0 & 66.7 & 66.7 & 33.3 & 33.3 & 33.3 \\
\hline Citrobacter freundii & 0.0 & 0.0 & 100 & 0.0 & 0.0 & 0.0 & 100 & 100 & 100 & 0.0 & 0.0 & 0.0 & 0.0 & 100 & 0.0 & 0.0 & 0.0 \\
\hline Chryseomonas luteola & 0.0 & 0.0 & 0.0 & 0.0 & 0.0 & 40.0 & 0.0 & 0.0 & 40.0 & 0.0 & 80.0 & 0.0 & 40.0 & 100 & 0.0 & 0.0 & 100 \\
\hline
\end{tabular}

AM-ampicillin; $\mathrm{AU}$-augumentin; $\mathrm{CH}$ - chloramphenicol; $\mathrm{CL}$-cloxacillin; $\mathrm{CL}_{2}$-clindamycin; $\mathrm{CO}$-clotrimazole; $\mathrm{CE}$-ceftriaxone; $\mathrm{CE}_{2}$ - $\mathrm{Cefuroxime}^{\mathrm{C}} \mathrm{CE}_{3}$ - ceftazidime; $\mathrm{CE}_{4}{ }^{-}$ ciprofloxacin; ER- erythromycin; GE- gentamycin; IM- imipenem; LE- levofloxacin; ME- meropenem; OF- ofloxacin; TE- tetracycline; VA- vancomycin

Antibiotics sensitivity pattern of the isolated organisms are shown in Table 3 . The Streptococcus spp had a high sensitivity to ceftriaxone (80\%) cefuroxime (90\%) and augumentin (80\%); moderate sensitivity to clindamycin (60\%), imipenem (60\%), ofloxacin (40\%) and low sensitivity to ampicillin (10\%), chloramphenicol (20\%), ceftazidime (20\%), erythromycin (30\%) and vancomycin (10\%). Likewise, the staphylococcus spp had no high sensitivity to any antibiotics. They had moderate sensitivity to augumentin (40\%), clindamycin (60\%), erythromycin (40\%), imipenem (60\%) and low sensitivity of $20 \%$ to cloxacillin, cefixime, gentamycin, ofloxacin and tetracycline. Escherichia spp were poorly responsive to virtually all antibiotic considered with moderate susceptibility to only augumentin (50\%), chloramphenicol (65\%) and imipenem (50\%). Pseudomonas spp had zero sensitivity to all except ceftazidime (100\%), ciprofloxacin (100\%), ofloxacin (100\%) and imipenem (100\%) while contrary to pseudomonas, Moraxella catarrhalis was $100 \%$ sensitive to all antibiotics except for cloxacillin, clindamycin, clotrimazole, cefuroxime, cefixime, levofloxacin, meropenem and vancomycin. Finally, Klebsiella spp had mixed sensitivity patterns with a $75 \%$ sensitivity to ceftriaxone and cefuroxime, $50 \%$ sensitivity to chloramphenicol and ofloxacin and a $25 \%$ sensitivity to augumentin, gentamycin and ofloxacin. Sensitivity pattern of other less common organisms isolated on blood culture are shown in Table 3.

Table 4 shows in summary the antibiotics coverage pattern of bacteria isolates based on their gram stain property. Imipenem had the strongest coverage with $92 \%$ against gram positive bacteria (GPB), $100 \%$ against gram negative bacteria (GNB) and an average coverage of $96 \%$. Ofloxacin performed relatively poorer with a $64 \%$ coverage of gram positive, $46 \%$ coverage for gram negative and $55 \%$ on average. Others included Augmentin, $62 \%$ (GPB), $50 \%$ (GNB) and average of $56 \%$; Vancomycin ( $60 \%$ for GPB. $75 \%$ for GNB and average of $67.5 \%$ ); Ceftriaxone ( 40 for GPB. $60 \%$ for GNB and average of $50 \%$ ); Cefotaxime (40\% for GPB, $60 \%$ for GNB and average of $50 \%$ ); Ceftazidime (7\% for GPB, $61 \%$ for GNB and average of $34 \%$ ); Cefuroxime ( $40 \%$ GPB. $53 \%$ for GNB and average of $46.5 \%$ ); and Gentamicin ( $20 \%$ for GPB, $42 \%$ for GNB and average of $31 \%$ ). Ampicillin was the weakest antibiotics with a $33 \%$ coverage for GPB, $0 \%$ coverage for GNB and an average coverage of $16.5 \%$. 
Table 4

Antimicrobial sensitivity pattern of blood culture isolates

against commonly available antibiotics in newborn

admitted to SCBU of the UATH between September 2013 to

April 2014

\begin{tabular}{|llll|}
\hline Antibiotics & Sensitivity & & \\
\hline & Gram positive & $\begin{array}{l}\text { Gram } \\
\text { negative }\end{array}$ & $\begin{array}{l}\text { Average } \\
\text { Sensitivity }\end{array}$ \\
\hline Imipenem & 92 & 100 & 96.0 \\
\hline Ofloxacin & 64 & 46 & 55.0 \\
\hline Augmentin & 62 & 50 & 56.0 \\
Vancomycin & 60 & 75 & 67.5 \\
\hline Ceftriaxone & 40 & 60 & 50.0 \\
\hline Cefotaxime & 40 & 60 & 50.0 \\
\hline Ceftazidime & 7 & 61 & 34.0 \\
\hline Cefuroxime & 40 & 53 & 46.5 \\
\hline Gentamicin & 20 & 42 & 31.0 \\
\hline Ampicillin & 33 & 0 & 16.5 \\
\hline
\end{tabular}

\section{Discussion}

Bacterial isolation rate of $13.6 \%$ was observed in this study, this is lower than what was reported in studies within and outside the country. In llorin, a bacteria isolation rate of $30 \%,{ }^{18}$ was reported and $34 \%$ in India. ${ }^{19}$ However, both the llorin and Indian studies excluded subjects with any prior exposure to antibiotics unlike this current study which included newborns with 24 hours or less antibiotic exposure. This could be responsible for the high isolation in the two referenced studies. There may be need for BACTEC use in more studies at various centres to validate its applicability in the tropics where antibiotics is readily available over the counter. ${ }^{9}$

Our study found that more than half $(53.9 \%$ ) of the bacteria isolates were Gram positive organisms, while Gram negative organisms made up $46.1 \%$. This pattern was found in Qatar ${ }^{20}$ where a predominance of Gram-positive bacteria was noted (66\%) and 16.2\% for Gram negative bacteria, in Karachi (54.1\%) ${ }^{21}$ for Gram positive bacteria and $45.9 \%$ for GNB. In Gwagwalada, a report of $58 \%$ for GPB and $42 \%$ for GNB. ${ }^{11}$ Some other studies also revealed preponderance of GPB over GNB. ${ }^{22,23}$ Unlike our study, other studies found a preponderance of GNB over GPB. Khetam et al ${ }^{21}$ reported $54.5 \%$ for GNB and $45.4 \%$ for GPB, while another study in Pakistan ${ }^{24}$ showed GNB of $54.6 \%$ and GPB of $45.4 \%$. However, Iregbu et al ${ }^{25}$ in Abuja, found equal occurrence of both Gram positive and negative organisms. These variations are possibly due to organisms acquired from the birth canal for the early onset sepsis and from the community for the late onset sepsis.

Enterococcus faecalis was the most common gram-positive organism isolated in this current study, while Escherichia coli were the commonest gram-negative organisms. This is a departure from findings from a similar study that was carried out in the unit 10 years earlier, where Staphylococcus aureus was found to be the commonest organism and Klebsiella was the leading Gram negative organism. ${ }^{11}$ This further corroborates the fact that, the organisms responsible for neonatal sepsis vary from time to time, even in the same unit, and possible changing pattern of neonatal sepsis. The Enterococcus faecalis isolated did not demonstrate multi-resistant pattern, whuch favours the possibility of a non-nosocomial source of the isolates, even though it is usually associated with nosocomial infection.

Staphylococcus aureus was the third commonest Gram-positive bacteria after Enterococcus specie and Streptococcus specie in the current study. Iregbu et $\mathrm{al}^{25}$ in Abuja, found Enterococcus faecalis as a major cause of neonatal sepsis second only to Staphylococcus aureus among the Gram-positive bacteria. It was also found to be the commonest Gram-positive bacteria causing neonatal sepsis in Accra, ${ }^{26}$ accounting for $14.4 \%$ of the organisms isolated. The Abuja study was a retrospective study, so some of the data may be incomplete. It could also be a pointer, to the emergence of Enterococcus, as an important cause of neonatal sepsis in Abuja. Similarly, Escherichia coli were the commonest GNB identified as causes of neonatal sepsis in studies within Nigeria and outside. $24,27-29,30,31$ It was also a leading cause of Gram-negative neonatal sepsis in some other studies, like the ones from Portharcourt ${ }^{24}$ and Ife. ${ }^{22}$

Klebsiella specie and Staphylococcus aureus were the third commonest Gram-negative bacteria and Gram-positive bacteria respectively in this study. This was a sharp departure from many studies both locally and internationally, where these organisms were identified as the leading causes of neonatal sepsis. ${ }^{11}$, $21,24,28,32-36$ The reason for this may not be immediately deduced, but it could be due to the method of isolation, as there is no reported study in the Northern part of the country where BACTEC machine was used for bacteria isolation yet. This could also be an emerging pattern of neonatal sepsis in the country, particularly in the North central region. Citrobacter spp accounted for $1.8 \%$ of neonatal sepsis in our study, which was comparable to the $3.1 \%$ reported in a study done by Ramesh et al. ${ }^{36}$ This organism was however not reported in other similar studies. ${ }^{11,18}$ 
Ella et al in Kaduna, ${ }^{37}$ reported similar antimicrobial sensitivity pattern of gram-positive organisms as reported in our study. Studies in India, ${ }^{23,38}$ reported $80 \%$ sensitivity to vancomycin, whereas Bode Thomas et al ${ }^{24}$ in Jos reported $67 \%$ sensitive of GPB to gentamicin. Iruegbu et al ${ }^{25}$ showed an $89 \%$ sensitivity of GPB to Augmentin. Studies in Calabar, Uganda and India, ${ }^{30,36,39}$ observed a high sensitivity to gentamicin. Studies in South West Nigeria, ${ }^{22,40}$ reported a high sensitivity to ofloxacin which is comparable with the finding of this study. The reason for low sensitivity of GPB to gentamicin in this study could be as a result of extensive use of gentamicin as empiric antibiotics in our neonatal unit. Possibly the bacteria are developing resistance to it. Also, the varied sensitivity pattern of Gram-positive organism across the country supports the theory that organisms associated with neonatal sepsis and their sensitivity varies from place to place and region to region. ${ }^{1,8}$ Use of empiric antibiotics as earlier stated, could be another reason why the sensitivity of these organisms to the antibiotics differs.

Gram-negative bacteria sensitivity pattern seen in this study is similar to the findings in Abuja ${ }^{25}$ and Baghdad, ${ }^{27}$ where $100 \%$ sensitivity of Gram-negative bacteria to imipenem and meropenem was reported. A good sensitivity of Gram-negative bacteria to cephalosporins, cefotaxime $86.7 \%$, ceftazidime $81.3 \%$ was reported in Sagamu. ${ }^{35}$ Mugalu et al in Uganda, ${ }^{39}$ reported a $94.1 \%$ sensitivity of Escherichia coli to ceftriaxone while Iregbu ${ }^{25}$ in $\mathrm{Abuja}$ observed $94 \%$ sensitivity to cefotaxime. Though much higher than what was found in this study, it shows that cephalosporins are very active against Gram negative bacteria. Therefore, caution must be applied in the use of these cephalosporins as empirical antibiotics, so resistance is not developed these important class of antibiotics as was reported for gentamycin in this study.

Limitation

One major limitation we had during this study was inability of some babies who presented over the weekend to be immediately screened for sepsis because the BACTEC laboratory was closed on weekends. This may have affected culture of organisms since their blood after collection had to be stored over the weekends.

\section{Conclusion}

Neonatal sepsis is prevalent in our setting. Enterococcus and streptococcus species were the commonest gram-positive organisms while Escherichia coli and Chryseomonas luteola were the commonest gram-negative organism. Imipenem had the widest coverage for both gram-positive and gram-negative organism. Due to poor sensitivity of gentamicin observed in this study, the current empiric antibiotic combination of Amoxicillin/Clavulanic acid and Gentamicin should be changed to Amoxicillin/Clavulanic acid and a third-generation cephalosporin such as Ceftriaxone and Cefotaxime. Lastly, even though, Imipenem had the widest coverage for bacteria isolated in study participants, its use should be reserved for serious infections to prevent resistance and minimize cost.

\section{List Of Abbreviations}

\begin{tabular}{|ll|}
\hline EOS & Early onset sepsis \\
\hline ESR & Erythrocyte sedimentation rate \\
\hline GBS & Group B Streptococcus \\
\hline GNB & Gram negative bacteria \\
\hline GPB & Gram positive bacteria \\
\hline LOS & Late onset sepsis \\
OFC & Occipito-frontal circumference \\
\hline SCBU & Special care baby unit \\
\hline UATH & University of Abuja Teaching Hospital \\
\hline WBC & White blood cells \\
\hline
\end{tabular}

\section{Declarations}

Ethics approval and consent to participate

Ethical clearance was obtained from the Medical Ethics Committee of the University of Abuja Teaching Hospital, Gwagwalada for review and approval was obtained before the commencement of the research (reference number FCT/UATH/HREC/PR/304). Written informed consent was obtained from a parent and/or guardian for all participants. Additionally, verbal consent was obtained for older children who were able to give approval to be enrolled in the study. Participation in the study was entirely voluntary, and no financial inducement whatsoever was involved. Participants were informed that voluntary withdrawal at any stage of interaction was guaranteed for them without any adverse effect to them. All information was handled with strict confidentiality.

Consent for publication

Not applicable

Availability of data and material 
The datasets used and/or analysed during the current study are available from the corresponding author on reasonable request.

Competing interests

The authors declare that they have no competing interests

Funding

Funding for this study was from equal contributions from all authors. No External funding was received for this study.

Authors' contributions

$\mathrm{OO}$ conceptualized the study and together with KIA and KEO developed the study methodology. ODIC analysed the study data. Result and discussion were written by ODIC KKI and OI. Supervision of data collection and laboratory sample analysis was done by OK and NI. KIA and KEO supervised the entire work. All authors read and approved the final manuscript.

Acknowledgements

We are also grateful to the highly dedicated staff nurses SCBU for their tireless efforts in some study related documentations and heroic efforts in salvaging sick babies.

\section{References}

1. Premalatha D.E, Mallikarjun K, Halesh L.H, Siddesh K.C, Prakash N. The bacterial profile and antibiogram of neonatal septicaemia in a tertiary care hospital. International Journal of recent trends in Science and technology. 2014;10: 451-55.

2. Leah Y, Rajam R. Assessing and applying evidence-based interventions at the community level in India as a model policy to reduce neonatal mortality rates in Nigeria. Available at www. jglobalhealth.org I - last accessed on $2^{\text {nd }}$ July 2015.

3. Bambala P Z, Vishnu B, Belgode N H, Thirunavukkarasu A B, Noyal M J. Neonatal sepsis in a tertiary care hospital in South India: bacteriological profile and antibiotic sensitivity pattern. Indian J Pediatr DOI 10.1007/s12098-010-0314-8.

4. Zaidi A K M, Ganatra H A, Syed S, Cousens S, Lee A C C, Black R et al. Effect of case management on neonatal mortality due to sepsis and pneumonia. BMC Pub HIth 2011; 11: 1-15.

5. Federal Ministry of Health. Saving newborn lives in Nigeria: Newborn health in the context of the Integrated Maternal, Newborn and Child Health Strategy. 2nd edition. Abuja: Federal Ministry of Health, Save the children, Jhpiego; 2011.

6. Child Mortality Estimates. Country-specific neonatal mortality rate Estimates generated by the UN Inter-agency Group for Child Mortality Estimation (UN IGME) in 2019. Downloaded from http://data.unicef.org

7. Osrin D, Vergnano S, Costello A. Serious bacterial infections in newborn infants in developing countries. Curr Opin Infect Dis 2004; 17:217-24.

8. Masood H R, Sadia K, Tooba, Sabah N, Sadaf S. Sepsis in infants: analysis of bacterial pathogens and their antibiotic susceptibility, a study at Government Tertiary Care Hospital, Karachi. J Dow Univ HIth Sc Karachi 2013; 7: 35-40.

9. Vergnano S, Sharland M, Kazembe P et al. Neonatal sepsis: an international perspective. Arch Dis Child Fetal Neonatal 2005; 90: 220-224.

10. Ogunlesi AT, Olusoga BO. Predictors of mortality in neonatal septicemia in an under-resourced setting. J Natl Med Assoc 2010; 102 : 915-21.

11. Olateju EK, Okechukwu AA, Mokuolu OA. Neonatal septicaemia at the University of Abuja Teaching Hospital, Gwagwalada, Nigeria. New Nig J Clin Res 2011; 1: 95-103.

12. Population distribution by Sex, State, LGA \& Senatorial district, available at population.gov.ng, last accessed on the $27^{\text {th }}$ May 2015 .

13. Lwanga SK, Lemeshow S. Sample size determination in health studies. WHO 1991: 1-30

14. New Ballard score. Available at ballardscore.com/files/Ballardscore_scoresheet.pdf, last accessed on $26^{\text {th }}$ May 2015.

15. Olusanya O. Okpere E, Ezimokhai M. The importance of Social class in voluntary fertility control in a developing country. W Afr J Med 1985; 4: $205-212$.

16. BACTEC instrumented blood culture; available at Becton,Dikinson and company-diagnostic system.com, last accessed on $18^{\text {th }}$ November 2014.

17. Clinical and Laboratory Standard Institute; Performance Standards for Antimicrobial Susceptibility Testing; Twenty second Informational Supplement. 2012 (M100 S22) 32:1- 139.

18. Mokuolu AO, Jiya N, Adesiyun 00. Neonatal septicaemia in Ilorin: bacterial pathogens and antibiotic sensitivity pattern. Afr J Med Sci 2002; 31 : 127-30.

19. Chacko B and Sohi I. Early onset neonatal sepsis. Indian journal of paediatrics; 2005; 72: 23-26.

20. Afif A, Samawal L, Abdul Rouf PV, Moza A, Sajjad R, Wessam El Kassim, et al. Incidence of bacterial isolates from blood culture in the neonatal intensive care unit of Tertiary Care Hospital. Int J Drug Dev and Res 2012; 4: 359-67.

21. Khetam HRA, Deia KK and Lamia A. The bacterial profile and c-reactive protein of suspected septic neonates admitted to the Al-Kadyemia teaching hospital. Internat J Recent Scientific Res 2013; 11: 1723-27.

22. Adejuyigbe E.A, Adeodu 0.0, Ako-nai K. A, Taiwo O, Owa J.A. Septicaemia in high risk neonates at a teaching hospital in lle-lfe, Nigeria. East Afr Med J 2001; 78: 540-43.

23. Tallur SS, Kasturi AV, Shobha D. Nadgir, Krishna BVS. Clinico-bacteriological study of neonatal septicemia in Hubli. Indian J Pediatr 2000; $67: 169$-74. 
24. Bode-Thomas F, Ikeh El, Pam SD, Ejeliogu EU. Current aetiology of neonatal sepsis in Jos University Teaching Hospital. Niger J Medicine: J National Assoc Resident Doctors Nigeria 2004; 13:130-135

25. Iregbu KC, Elegba OY, Babaniyi IB. Bacteriological profile of neonatal septicaemia in a tertiary hospital in Nigeria. Afr HIth Sc 2006; 6: 151-4.

26. Anyebuno M, Newman M. Common causes of neonatal bacteraemia in Accra, Ghana. East Afr Med J 1995; 72: 805-8.

27. Tosson AM, Speer CP. Microbial pathogens causative of neonatal sepsis in Arabic countries. J Matern Fetal Neonatal Med 2011; 24: 990-4.

28. Awoala WB and Tabansi PN. Clinico-bacteriological profile of early and late onset sepsis in a tertiary hospital in Nigeria. J Medicine Med Sc 2012; 3: 10711.

29. Neonatal sepsis: Self-learning packet 2004. Available at www.orlandohealth.com/pdf folder/neonatal sepsis.pdf, last accessed on $25^{\text {th }}$ May 2015.

30. Antia-Obong OE, Utsalo SJ, Udo JJ, Udo KT. Neonatal septicaemia in Calabar, Nigeria. Central Afr J Med 1992; 38 : 161-5.

31. Ghiorghis B. Neonatal sepsis in Addis Ababa, Ethiopia: a review of 151 bacteremic neonates. Ethiop Med Journal $1997 ; 35$ : 169-76.

32. Murli LM, Jyoti G, Ruchika S, Aruna S. Rapid culture of Mycobacterium tuberculosis on blood agar in resource limited setting. Dan Med. Bull. 2009; 20810.

33. Ojewumi TK, Ojewumi JS. Trends in infant and child mortality in Nigeria: a wakeup call assessment for intervention towards achieving the 2015 MDGs. SC $J$ Sociol Anthropol 2012;

34. Stoll JB. Infections of the neonatal infant. In: Kliegman RM, Behrman RE, Jenson HB, Stanton BF (editors). Nelson textbook of Pediatrics.18 ${ }^{\text {th }}$ ed. Philadelphia: Saunders Elsevier; 2007; p. 784-803.

35. Ogunlesi TA, Ogunfowora OB, Osinupebi O, Olanrewaju DM. Changing trends in newborn sepsis in Sagamu, Nigeria: bacterial aetiology, risk factors and antibiotic susceptibility. J Paediatr Child HIth 2011; 47: 5-11.

36. Ramesh BY, Leslie ESL, Vandana KE. Bacterial isolates of early-onset neonatal sepsis and their antibiotic susceptibility pattern between 1998 and 2004 : an audit from a center in India. Ital J Pediatr 2011; 37: 32

37. Ella EE, Ahmad AA, Ogala WN, Umoh VJ Aliyu-Zubair R. Bacteriology and sensitivity profile bacterial agents responsible for neonatal septicaemia in a Tertiary Hospital of Kaduna Metropolis. J pure applied microbio/ 2008; 2: 103-8.

38. Ghanshyam DK, Ramachandran VG, Gupta P. Bacteriological analysis of blood culture isolates from neonates in a Tertiary Care Hospital in India. $J$ Health Popul Nutr 2002; 20: 343-47.

39. Mugalu J, Nakakeeto MK, Kiguli S, Kaddu - Mulindwa DH. Aetiology, risk factors and immediate outcome of bacteriologically confirmed neonatal septicaemia in Mulago Hospital, Uganda. Afr HIth Sc 2006; 6: 120-6.

40. Awoniyi O, Udo SJ, Oguntibeju 00. An epidemiological survey of neonatal sepsis in a hospital in western Nigeria. Afr J Microbiol Res 2009; 3: 385-9.

\section{Figures}




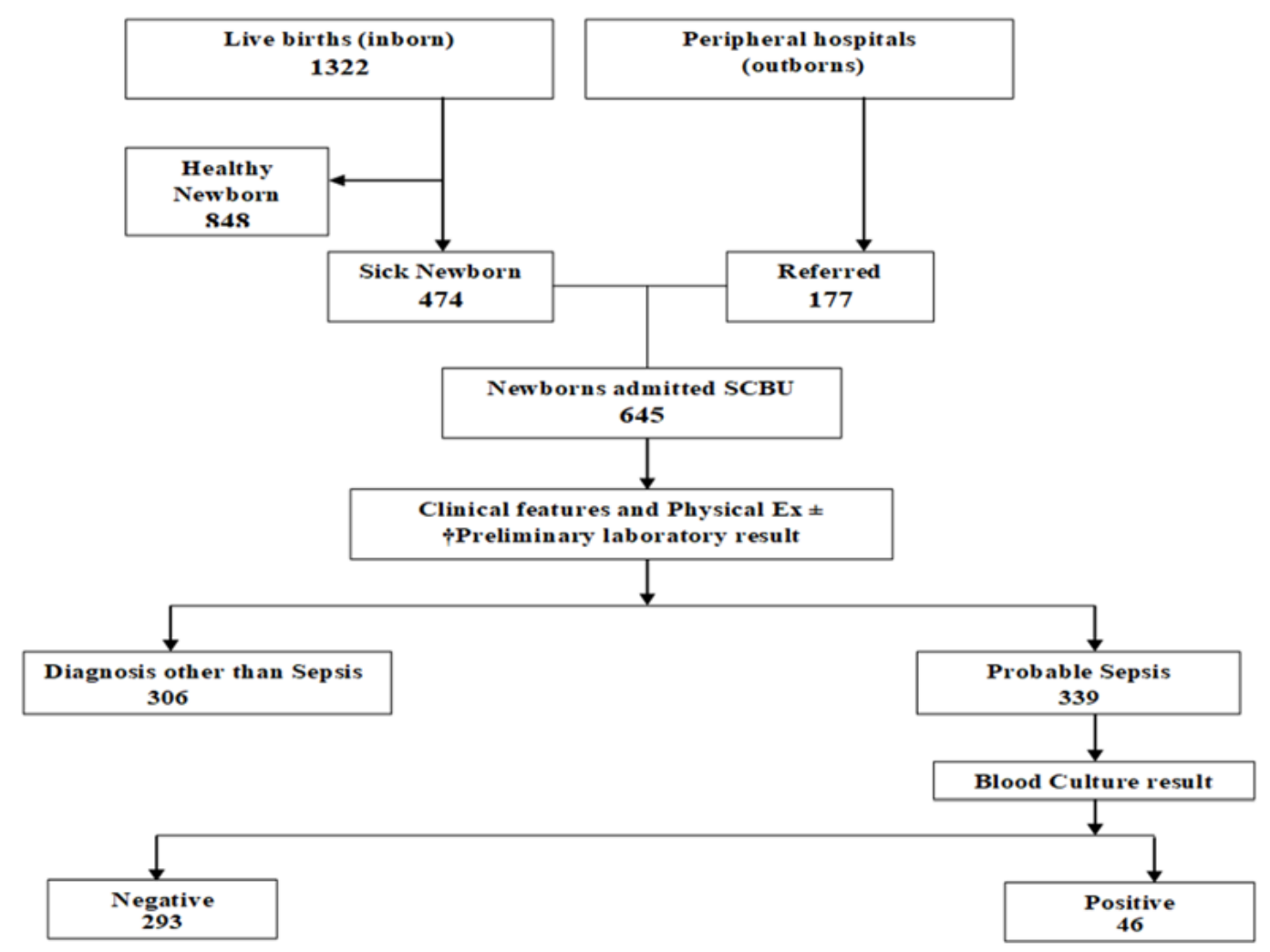

Figure 1

Overview of recruitment processes of surveyed newborns in the SCBU between September 2013 to April 2014 (†Complete blood count, urinalysis and gram stain)

\section{Supplementary Files}

This is a list of supplementary files associated with this preprint. Click to download.

- supplementaryfile.docx 